\title{
Corela
}

Cognition, représentation, langage

$12-2 \mid 2014$

Vol. $12, n^{\circ} 2$

\section{Les discours institutionnels en confrontation}

Contribution à l'analyse des discours institutionnels et politiques

\section{Silvia Modena}

\section{(2) OpenEdition}

Journals

Édition électronique

URL : http://journals.openedition.org/corela/3611

DOI : $10.4000 /$ corela.3611

ISSN : 1638-573X

Éditeur

Cercle linguistique du Centre et de l'Ouest - CerLICO

Référence électronique

Silvia Modena, "Les discours institutionnels en confrontation », Corela [En ligne], 12-2 | 2014, mis en ligne le 15 décembre 2014, consulté le 22 septembre 2020. URL : http://journals.openedition.org/ corela/3611; DOI : https://doi.org/10.4000/corela.3611

Ce document a été généré automatiquement le 22 septembre 2020.

\section{cc) () (ㅇ)}

Corela - cognition, représentation, langage est mis à disposition selon les termes de la licence Creative Commons Attribution - Pas d'Utilisation Commerciale - Partage dans les Mêmes Conditions 4.0 International. 


\title{
Les discours institutionnels en confrontation
}

\author{
Contribution à l'analyse des discours institutionnels et politiques
}

\author{
Silvia Modena
}

1 En affirmant le lien indissociable qui soude la problématique des discours institutionnels et la genèse de l'analyse du discours, Julien Longhi et Georges-Elia Sarfati présentent l'ouvrage Les discours institutionnels en confrontation - Contribution à l'analyse des discours institutionnels et politiques publié chez L'Harmattan. Ce volume est organisé suivant deux perspectives différentes mais tout à fait complémentaires : d'une part, les auteurs qui ont contribué à cette publication se penchent sur des corpus institutionnels spécifiques, de l'autre une théorisation de toute institution discursive est ponctuellement proposée. Les contributions qui composent ce volume ont été recueillies dans la perspective d'une analyse renouvelée des discours institutionnels et politiques en essayant d'articuler les méthodes d'analyse et le choix des observables.

2 Le premier chapitre, occupé par la contribution de Georges-Elia Sarfati (L'emprise du sens: note sur les conditions théoriques et les enjeux de l'analyse du discours institutionnel), associe l'analyse du discours et la philosophie dans l'effort de restituer à l'analyse du discours institutionnel sa propre autonomie épistémologique. Plus spécifiquement, l'auteur met l'accent sur les notions de sens (commun) et d'institution (de sens). Décrivant les traitements théoriques qui ont permis d'aboutir à une "conception institutionnelle» du sens commun (mécanismes discursifs et sociaux), l'auteur en illustre le cadre organisationnel et sémiotique. Pour ce qui concerne les institutions de sens, des typologies sont schématisées afin de classifier les productions discursives des « sujets-acteurs » qui en sont partie prenante. A ce propos, l'auteur ouvre une réflexion sur la subjectivité en relation aux instituions de sens (insertion et inscription). En proposant un scénario possible pour de nouvelles recherches sur le sens commun et l'institution de sens, l'auteur introduit le concept de « lien social». Si la rhétorique (les lieux du discours de Aristote), la pragmatique de O. Ducrot et la nouvelle rhétorique de C. Perelman ont été les piliers théoriques de l'élaboration conceptuelle du sens 
commun, c'est l'étude de l'institution de sens qui, à travers le lien social, explicite les discours qu'elle génère.

3 Le deuxième chapitre est dédié à l'étude de la formule "arts premiers ». L'article $L a$ formule «arts premiers»: désignations et ajustement institutionnels $d u$ "Musée du Quai Branly» de Laetitia Grosjean aborde cette notion en tant que rupture: en effet, les polémiques précédant l'institutionnalisation du musée, suscitées par le choix de l'adjectif " premiers » a permis l'étude de la construction d'un discours institutionnel pendant la gestation du musée. Le corpus d'étude se compose d'articles de presse quotidienne repérés et sélectionnés en accord avec la notion de moment discursif proposé par S. Moirand. L'aspect chronologique des données recueillies est ensuite soudé à la notion de sens commun de G.-E. Sarfati (topique sociale et topique configurationnelle) mobilisée afin d'étudier le poids désignatif de l'institution muséale. Tout en retravaillant la distinction de J. Guilhaumou concernant les types d'événements (é. linguistique, é. discursif et récit d'é.), l'auteur analyse la formule « arts premiers » à travers son hétérogénéité désignative en diachronie. Si les critiques à cette dénomination relèvent des opinions des acteurs du débat, une sorte d'objectivisation du nom géographique du musée s'impose successivement. Le Musée est ainsi lié au " quai Branly " même si, selon l'auteur, la formule "arts premiers » continue à être employé dans l'usage commun. L'auteur souligne, en conclusion, l'ajustement discursif perpétuel de l'institution par rapport au nom du musée.

4 L'étude de Carine Duteil (Le traitement des crises sanitaires: entre parole institutionnelle et discours médiatiques) présente le traitement médiatique de deux crises sanitaires: le frelon asiatique à pattes jaunes et l'affaire de la bactérie tueuse E. Coli. Les deux corpus sont constitués respectivement à partir d'articles tirés des quotidiens Le Monde (Hyperbase) et Sud Ouest. L'auteur, dans le sillage des travaux de P. Charaudeau sur la vulgarisation médiatique et ses effets de dramatisation, concentre son attention sur la mise en scène médiatique de ces événements. L'analyste pose que les événements de ce type mobilisent une certaine mémoire interdiscursive qui fait levier sur la composante pathémique du discours alarmiste et catastrophique. Cette posture interprétative est le fruit de la combinatoire entre le poids interprétatif des titres et les images alarmantes publiées par les quotidiens analysés. L'auteur souligne également que la dimension politique de la crise pourrait être le fruit d'une communication institutionnelle mal maitrisée car le traitement de ces crises sanitaires concerne différentes " couches » médiatiques. Autrement dit, des crises agricoles de ce genre déclenchent des effets économiques, politiques et diplomatiques de telle façon qu'il se produit un glissement de l'événement: du milieu médical-scientifique au milieu politicoéconomique.

5 Dans le quatrième chapitre Silvia Nugara présente son étude Analyser le discours institutionnel international: le cas des documents du Conseil de l'Europe sur la violence domestique envers les femmes. L'auteure, qui conçoit le discours en tant que « pratique de constitution et de légitimation des acteurs publics ", adopte une méthodologie croisant l'anthropologie du discours institutionnel (C. Oger et C. Ollivier-Yaniv) et l'analyse du discours institutionnel (C. Oger et A. Krieg-Planque).

6 Le traitement de la « violence domestique » est identifié dans l'institution du Conseil de l'Europe représenté par un corpus de 90 documents rédigés à partir de 1985 jusqu'à la Convention d'Istanbul (2011). La stratification discursive des productions du Conseil de l'Europe crée un ensemble hétérogène de documents qui font appel à deux typologies 
discursives différentes mais agencées par le rapport qu'elles entretiennent avec l'institution. A ce propos, l'auteur rappelle le clivage existant d'une part entre le discours politique et le discours institutionnel (D. Maingueneau), de l'autre, entre le discours institutionnel et le discours instituant (C. Oger et C. Ollivier-Yaniv). Selon une perspective énonciative, l'auteur définit le discours du Conseil de l'Europe en tant que discours sans opposants. Autrement dit, les documents produits par cette institution ne doivent que susciter le consensus des gouvernements. L'analyse, menée en suivant le parcours nominatif du syntagme "violence domestique » et la mobilisation des Etats, amène l'auteure à définir le discours de l'institution en tant que discours dense de formes dénominatives indéterminées qui garantissent un certain "monopole énonciatif ».

7 Oceane Hô-Dình et Mathieu Valette traitent la notion d'autorité par rapport à Internet dans leur contribution: Textes institutionnels et textes informels issus du web social. Analyse différentielle des discours de prévention $d u$ VIH en français et en vietnamien. Les problématiques sanitaires causées par le VIH en France et au Vietnam sont observées à travers les notions de stabilité/instabilité dans les discours institutionnels et informels. Le corpus se compose de deux sous-corpus institutionnels (Sida Info Services français et HIV on line vietnamien) et de deux sous-corpus informels (messages forums). Deux outils lexicométriques ont permis aux auteurs de traiter ces données : pour la langue vietnamienne le logiciel vnTokenizer, pour le français TreeTagger/TXM. En partant du constat que le discours institutionnel traitant ces problématiques sanitaires est insuffisant et lacunaire, les auteurs repèrent, dans les forums de discussion, une sorte de stabilité liée à la réappropriation du discours institutionnel. Si le discours institutionnel génère catégorisation, abstraction et généralisation, le discours informel est focalisé autour des pratiques concrètes. A ce propos, une autre opposition est décelée à travers l'étude des pronoms personnels : l'existence d'une « zone anthropique distale»vs «zone anthropique identitaire». En d'autres termes, le traitement de la prévention du VIH incarne deux perspectives différentes : d'un côté l' «onthos » du discours institutionnel de l'autre la « praxis » du discours informel.

8 Le dernier chapitre accueille la contribution de Julien Longhi, L'hybridation du discours institutionnel à l'épreuve du numérique: renouvellement et reconfiguration de la parole institutionnelle. L'auteur étudie deux moments discursifs distincts connexes à deux discours institutionnels du Premier Ministre Jean-Marc Ayrault: le vote de la loi du « mariage pour tous » et les déclarations à propos de la nomination du couple banlieue/ quartier. Pour ce qui concerne la méthodologie adoptée, l'auteur mobilise un dispositif qui croise la pragmatique linguistique et la sémantique du discours. Cette perspective lui permet de "considérer le matériau discursif comme constitution du monde social [...], réel ». L'institution ainsi que la communauté de sens, selon les travaux de G.-E. Sarfati, permettent à l'auteur d'aborder la composition disparate du discours institutionnel au contact du numérique. Pour la présente étude, la dissociation dénominative entre l'homme et l'institution se manifeste à travers l'étude de deux comptes Twitter: Matignon et le nom du Premier Ministre. Le numérique s'enchevêtre donc avec la subjectivité du discours institutionnel. Trois comptes Twitter peuvent ainsi être décrits grâce aux notions de canon (@Matignon), vulgate (@jeanmarcayrault/@najatbv) et doxa (@internautes) élaborées par G.-E. Sarfati. Le traitement du vote "mariage pour tous » et de la nomination des banlieues/quartiers ne fait que confirmer l'hypothèse d'une "mutation que le numérique apporte au discours institutionnel». Ces mutations permettent aux discours d'activer un certain 
nombre de renvois interdiscursifs ainsi que de produire une forte dissociation énonciative rendue possible, en particulier, par Twitter.

\section{AUTEURS}

\section{SILVIA MODENA}

Lingua e Linguistica Francese 1, Scuola di Lingue e Letterature, Traduzione e Interpretazione, Dipartimento di Lingue, Letterature e Culture Moderne - LILEC, Bologna, Italia 